\title{
PVDF based Piezoelectric Nanogenerator as a new kind of device for generating power from renewable resources
}

\author{
Binoy Bera ${ }^{1}$, Madhumita Das Sarkar ${ }^{2}$ \\ ${ }^{1,2}$ Department of Computer Science and Engineering \\ West Bengal University of Technology, Kolkata - 700064, INDIA
}

\begin{abstract}
Polyvinylidene fluoride, polyvinylidene difluoride or simply PVDF is a chemically stable thermoplastic fluoropolymer synthesized by the polymerization of vinylidene difluoride. Polyvinylidene fluoride $(P V D F)$ is a piezoelectric polymer material which gives voltage as output when pressure or mechanical force applied on it. Based on this properties it is used in different sensing technology. There are different renewable sources present in environment like ocean waves, rain and wind. PVDF based piezoelectric nanogenerator can be a novel new kind of device which can generate power from all these renewable resources. In this article nanofiber based PVDF nanognerator has been prepared.Taken the output from finger pressure. Output power is mainly depend on the crystallinity and electroactive phase of PVDF . A brief idea about the improvement of crystallinity and electroactive phase is also described here.
\end{abstract}

Keywords: Polyvinylidene fluoride, piezoelectricity, electroactive phase, nanogenerator, energy.

\section{Introduction}

Today energy is a great concern in the world. It can be of different types like renewable energy and non renewable energy depending on the sources. Non renewable energy are comes from fossil fuel, coal, petroleum, and natural gas. Some day it will come to an end. So the renewable energy is very important. Renewable energy is the energy which is collected from renewable sources like wind, rain, ocean waves, sunlight. Solar cell is a device which can convert solar energy (sunlight is the source) into electricity. Generally it generates high power like megawatt or kilowatt. It is also important to convert energy from other renewable sources like wind, ocean waves, rain. A piezoelectric nanogenerator is a device which can convert mechanical energy into electrical energy. It can generate power from wind, ocean waves and rain also. Some piezoelectric material like zinc oxide nanowire, barium titanate, lead zirconate titanate, barium zirconate titanate, polyvinylidene fluoride etc. are used to make such kind of devices. Some of them are not flexible and some are flexible. But it is possible to make flexible nanogenerator from non flexible material by growing it on a flexible substrate. Some material are toxic and some are non toxic. Among all piezoelectric material polyvinylidene fluoride (PVDF) is suitable choice for making piezoelectric nanogenerator. It has several advantage like flexible, non toxic, biocompatiable, high piezoelectric coefficient. PVDF has four crystalline phase like $\alpha, \beta, \gamma$ and $\delta^{[2-8]}$ depending on chain conformation. Among them $\alpha$ is non polar phase. It does not show piezoelectric property. $\beta, \gamma$ and $\delta$ are polar phase, so PVDF fiber/film containing $\beta, \gamma$ and $\delta$ phase shows piezoelectricity in it. Among these three polar phase $\beta, \gamma$ phase can be generated by using several process like metal nanoparticle doping, 2D metal dichalcogenides ${ }^{[1-43]}$ hydrated salt doping, electrospinning process etc.. If we apply mechanical force/pressure/vibration on this nanogenerator, it will generate power in microwatt ranges. So PVDF based nanogenerator is a new kind of device for storing energy from renewable sources of environment.

\subsection{Materials}

\section{PVDF Fiber Based Sensor Preparation}

Poly (vinylidene fluoride ) $(\mathrm{PVDF}$ ) pellets ( $\overline{\mathrm{M}} \mathrm{w} \approx 275$ 000, Sigma-Aldrich, USA), N, N-dimethylformamide (DMF), acetone (Merck Chemical, India).

\subsection{Electrospinning sample preparation}

For the preparation of electrospinning ${ }^{[44]}$ sample, first we prepare 12wt\% (w/v) PVDF - DMF solution . Kept this solution on magnetic stirrer for a definite time. Then added $4 \mathrm{ml}$ of acetone with $6 \mathrm{ml}$ PVDF - DMF solution and stirred (30 minutes) by magnetic stirrer for final solution $(10 \mathrm{ml})$ of electrospinning process. Then we load this samples in syringe $(20 \mathrm{ml})$ for electrospinning process.

\subsection{Preparation of PVDF fiber}

For making PVDF fiber electrospinning process is used here. Where, Solution infuse rate, Syringe to collector distance, Voltage parameters are fixed. Electrospinning fiber has been collected in aluminium foil. In figure 1 it is shown the formation of nanofiber by electrospinning process. 


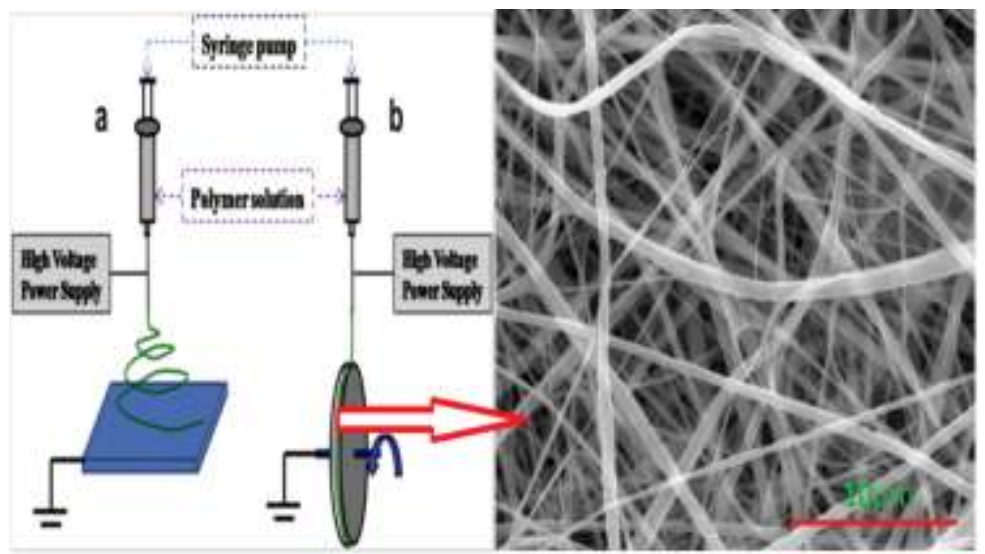

Fig.1 Schematic illustration of two(a and b) basic setup for electrospinning process. The right side shows a typical SEM image of the PVDF nanofibrous membranes deposited on the collector.

\subsection{Nanogenerator preparation}

Now electrospinning fiber has been seperated from aluminium foil. For the nanogenerator preparation , we use silver fabric as electrodes. In figure 2 it is shown how electrode has been placed on the both side of Electrospinning fiber.

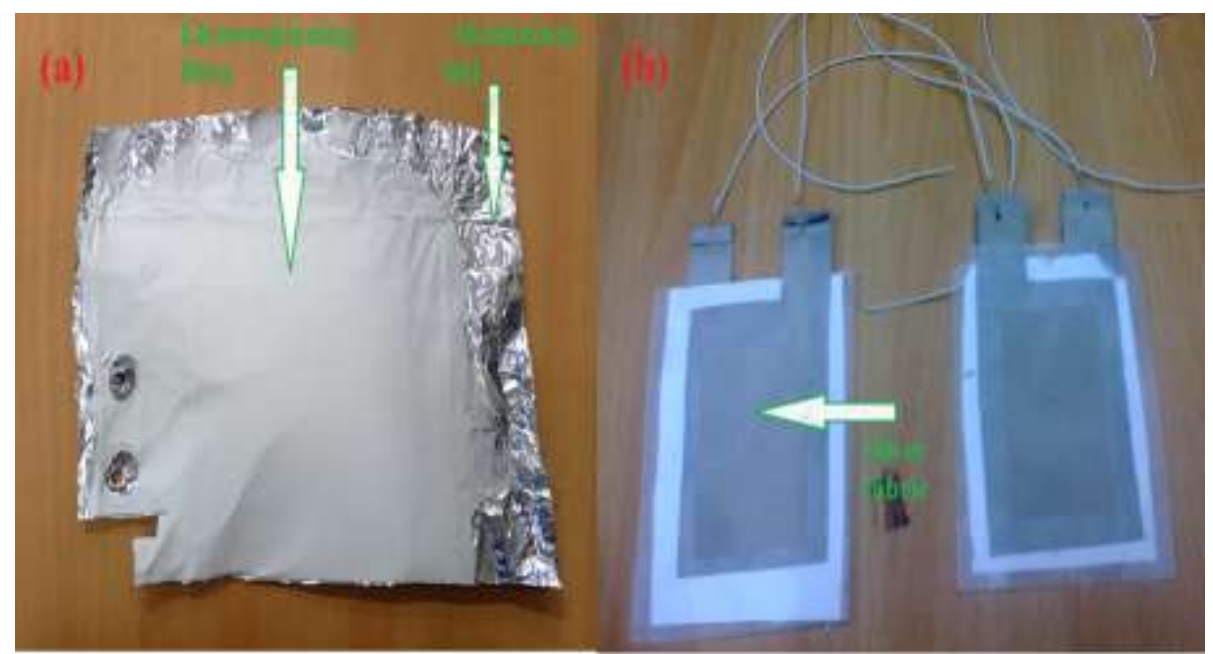

Fig.2 (a) Electrospinning fiber. (b) electrospinning fiber based nanogenerator.

\subsection{Output from nanogenerator}
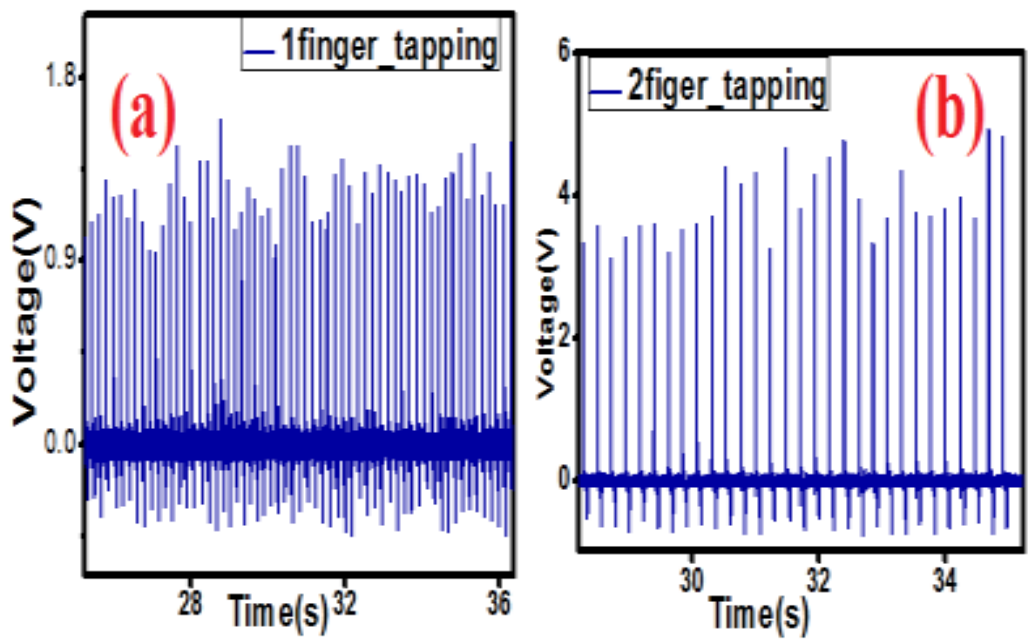

Fig.3 output voltage with (a) 1 finger tapping. (b) 2 finger tapping. 
Above figure shows sensing properties of PVDF material. So we can see that PVDF based nanogenerator can sense the pressure/force and it generates voltage as output. Similarly it can also generates voltage or power from previously mentioned renewable sources (ocean waves, wind energy, rain).

\section{How Output Power Can Be Improved}

Now the output power is dependent on the piezoelectric property of PVDF. Performance of PVDF material based sensor ${ }^{[18-57]}$ depends on the electroactive phase and crystallinity of this material and how quickly it can be achieved. There are several methods to obtain electroactive phases in PVDF .like mechanical stretching, poling, casting from solutions, spin coating, etc. Many researcher have also attempted to nucleate the electroactive phase within PVDF and improve the performance of the NG based sensor by incorporating graphene, gold nanoparticle, silver nanoparticle, nanoclay, CNTs, ${ }^{[45]}$ PMMA-RGO composite, and can be possible to make it ultrasensitive . Due to unique mechanical, chemical, electrical and/or optical properties of these material, electroactive phase of PVDF can be easily accessable. Electrical output of PVDF material based sensor depend on its crystallinity also.

For PVDF material containing $\alpha-, \beta$ - and $\gamma$ - phases the relative electroactive phase fraction ( $\mathrm{F}_{\mathrm{EA}}$ including, $\beta$ and $\gamma$-phases ) can be calculated using the following equation.

$\mathrm{F}_{\mathrm{EA}}=\frac{\mathrm{A}_{\mathrm{EA}}}{\left(\frac{\mathrm{K}_{840}}{\mathrm{~K}_{764}}\right) \mathrm{A}_{764}+\mathrm{A}_{\mathrm{EA}}} \times 100 \%$

where $\mathrm{A}_{\mathrm{EA}}$ and $\mathrm{A}_{764}$ are the absorbance intensities at 840 and $764 \mathrm{~cm}^{-1}$ respectively, and $\mathrm{K}_{841}\left(7.7 \times 10^{4} \mathrm{~cm}^{2}\right.$ $\left.\mathrm{mol}^{-1}\right)$ and $\mathrm{K}_{764}\left(6.1 \times 10^{4} \mathrm{~cm}^{2} \mathrm{~mol}^{-1}\right)$ are the absorption coefficients at the respective wavenumbers.

The total degree of the crystallinity $\left(\chi_{c_{t}}\right)$ is calculated by curve deconvolution methodology using equation 2 :

$\chi_{c t}=\frac{\sum A_{c r}}{\sum A_{c r}+\sum A_{a m r}} \times 100 \%$

Where $\Sigma \mathrm{A}_{\text {cr }}$ and $\Sigma \mathrm{A}_{\text {amr }}$ are the summation of the integral area of the crystalline peaks and the amorphous respectively. The degrees of $\beta$ - crystallinity and $\gamma$-crystallinity are calculated by the following equations:

$\chi_{c_{\beta}}=\chi_{c_{t}} \times \frac{\sum A_{\beta}}{\sum A_{\beta}+\sum A_{\gamma}} \%$

$\chi_{c_{\gamma}}=\chi_{c_{t}} \times \frac{\sum A_{\gamma}}{\sum A_{\beta}+\sum A_{\gamma}} \%$

Where $\Sigma \mathrm{A}_{\beta}$ and $\Sigma \mathrm{A}_{\gamma}$ indicate the total integral area from $\beta$ - and $\gamma$-crystalline phase peaks, respectively.

\section{Conclusion}

In this study PVDF based nanogenerator has been prepared. Mainly the output performance of this nanognerator depend on the electroactive phase and crystallinity of PVDF material. This kind of nanogenerator can be placed in anywhere where wind is blowing very high or even in lower portion of ships where ocean wave will struck the nanogenerator and it will generates voltage. Even in a rainy day, it can be placed on the roof or under the umbrella so that the power can be generate from rain drops. Such this way PVDF based piezoelectric nanogenerator can be a suitable device for generating power from all these renewable resources. Today the devices are becoming small like micro or nano size. Self powered nano or micro devices need power in the range of nano or micro watt. PVDF based nanogenerator generates power in nanowatt or microwatt ranges. So this nanogenerator is also useful for powering self powered micro or nano devices.

\section{Acknowledgements}

Binoy Bera would like to thank Dr. Madhumita Das Sarkar for her constant support, inspiration and guidance. I am also thankful to Dr. Dipankar Mandal of Department of Physics, Jadavpur University for giving me the opportunity to use his valuable instrument and laboratory. Binoy Bera wants to thank TEQIPphaseII,MAKAUT(Refno:09.02/Regis/Appnt.4/5) for providing him the fellowship.

\section{References}

[1.] S. Khan, S. Tinku, R. S. Dahiya, and L. Lorenzelli, Flexible Tactile Sensors using Screen Printed P(VDF-TrFE) and MWCNT/PDMS Composites, Sensors Journal, IEEE, vol. PP, pp. 1-1, 2014.

[2.] A.J. Lovinger, Developments in Crystalline Polymers, Vol. 1, (Ed. D.C. Basset, London, Elsevier Applied Science, 1982)

[3.] S. Lanceros-Mendez, J.F. Mano, A.M. Costa and V.H. Schmidt, J. Macrom, $\alpha$ - to - $\beta$ Transformation on PVDF Films Obtained by Uniaxial Stretch, Sci.- Phys. Vol. B40 (2001), p. 517

[4.] S. Laceros-Mendez, M.V. Moreira, J.F. Mano, V.H. Schmidt and G. Bohannan, Electrical and Microstructural Changes of $\beta$-PVDF under Uniaxial Stress Studied by Scanning Force Microscopy, Ferroelectrics Vol 273 (2002), p. 15 
[5.] A. Salimi, A. A. Yousefi, FTIR studies of $\beta$-phase crystal formation in stretched PVDF films. -Polymer Testing, Polymer Testing 22 (2003) p699-704.

[6.] K. Matsushige, K Nagata, S. Imada and T. Takemura, Effect of Mechanical Treatment Temperature on Electrical Properties and Crystallite Size of PVDF Film, Polymer (1980) Vol. 21, 1391-1397.

[7.] R. Gregorio Jr, E. M. Ueno, Effect of Solution Concentration on the Electrospray/Electrospinning Transition and on the Crystalline Phase of PVDF, Journal of Materials Science 34 (1999), 4489-4500.

[8.] R. Gregorio Jr, Marcelo Cestari, Effect of crystallization temperature on the crystalline phase content and morphology of poly(vinylidene fluoride), Journal of Polymer Science: Part B: Polymer Physics Vol 32 (1994), 859-870.

[9.] Novoselov, K. S.; Geim, A. K.; Morozov, S. V.; Jiang, D.; Zhang, Y.; Dubonos, S. V.; Grigorieva, I. V.; Firsov, A. A. Electric Field Effect in Atomically Thin Carbon Films, Science 2004, 306, 666- 669.

[10.] Schwierz, F. Nanomaterials: Graphene rolls off the press, Nature Nanotechnol. 2010, 5, 487- 496

[11.] Dean, C. R.; Young, A. F.; Meric, I.; Lee, C.; Wang, L.; Sorgenfrei, S.; Watanabe, K.; Taniguchi, T.; Kim, P.; Shepard K. L. et al. Boron nitride substrates for high-quality graphene electronics, Nature Nanotech. 2010, 5, 722- 726.

[12.] Novoselov, K. S.; Jiang, D.; Schedin, F.; Booth, T. J.; Khotkevich, V. V.; Morozov, S. V.; Geim, A. K. Two-dimensional atomic crystals, P. Natl. Acad. Sci. USA 2005, 102, 10451- 10453.

[13.] Radisavljevic, B.; Radenovic, A.; Brivio, J.; Giacometti, V.; Kis, A. Single-layer MoS2 transistors, Nature Nanotechnol. 2011, 6, 147- 150.

[14.] Fang, H.; Chuang, S; Chang, T. C.; Takei, K.; Takahashi, T.; Javey, A. High-performance single layered WSe 2 p-FETs with chemically doped contacts, Nano Lett. 2012, 12, 3788 - 3792.

[15.] Liu, W.; Kang, J.; Sarkar, D.; Khatami, Y.; Jena, D.; Banerjee, K. Role of metal contacts in designing high-performance monolayer n-type WSe2 field effect transistors, Nano Lett. 2013, 13, 1983- 1990.

[16.] Friend, R. H.; Yo_e, A. D. Electronic properties of intercalation complexes of the transition metal dichalcogenides, Adv. Phys. 1987, 36, 1- 94 .

[17.] Thurston, T. R.; Wilcoxon, Molybdenum Sulfide: Catalytic Properties in Nanoclusters , J. P. J. Phys. Chem. B $1999,103,11-17$.

[18.] Abrams, B. L.; Wilcoxon, J. P. MoS2 Nanoribbon Transistors: Transition from Depletion-mode to Enhancement-mode by Channel Width Trimming, Crit. Rev. Solid State Mater. Sci. 2005, 30, 153- 182.

[19.] Wilcoxon, J. P.; Newcomer, P. P.; Samara, G. A. Two-dimensional MoS2 as a new material for electronic devices J. Appl. Phys. 1997, 81, 7934- 7944.

[20.] Tenne, R.; Redlich, Recent progress in the research of inorganic fullerene-like nanoparticles and inorganic nanotubes, M. Chem. Soc. Rev. 2010, 39, 1423- 1434.

[21.] Mak, K. F.; Lee, C.; Hone, J.; Shan, J.; Heinz, T. F. Atomically Thin MoS2: A New Direct-Gap Semiconductor, Phys. Rev Lett. 2010, 105, 136805

[22.] Matte, H. S. S. R.; Gomathi, A.; Manna, A. K.; Late, D. J.; Datta, R.; Pati, S. K.; Rao, C. N. R. Angew. Chemically Exfoliated $\mathrm{MoS}_{2}$ as Near-Infrared Photothermal Agents, Chem. Int. Ed. 2010, 49, 4059- 4062

[23.] Lee, C.; Li, Q.; Kalb, W.; Liu, X. Z.; Berger, H.; Capick, R. W.; Hone, J. Frictional characteristics of atomically thin sheets, Science 2010, 328, 76- 79

[24.] Kam, K. K.; Parkinson, B. A. . Detailed Photocurrent Spectroscopy of the Semiconducting Group Vib Transition Metal Dichalcogenides. J. Phys. Chem. 1982, 86, 463- 467.

[25.] Chhowalla, M.; Shin, H. S.; Eda, G.; Li, L. J.; Loh, K. P.; Zhang, H. The chemistry of two-dimensional layered transition metal dichalcogenide nanosheets, Nature Chemistry 2013, 5, 263- 275.

[26.] Splendiani, A.; Sun, L.; Zhang, Y.; Li, T.; Kim, J.; Chim, C. Y.; Galli, G.; Wang, F. Emerging photoluminescence in monolayer MoS2, Nano Lett. 2010, 10, 1271- 1275.

[27.] Lebegue, S.; Eriksson, O. Electronic structure of two-dimensional crystals from ab initio theory, Phys. Rev. B 2009, $79,115409$.

[28.] Kuc, A.; Zibouche, N.; Heine, T. Influence of quantum confinement on the electronic structure of the transition metal sulfide TS2, Phys. Rev. B 2011, 83, 245213.

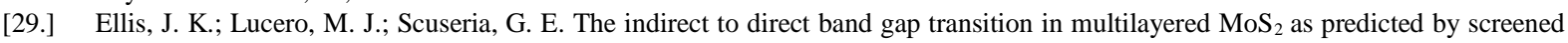
hybrid density functional theory, Appl. Phys. Lett. 2011, 99, 261908.

[30.] Kumar, A.; Ahluwalia, P. K., Electronic structure of transition metal dichalcogenides monolayers $1 \mathrm{H}-\mathrm{MX} 2(\mathrm{M}=\mathrm{Mo}$, W; $\mathrm{X}=\mathrm{S}$, $\mathrm{Se}, \mathrm{Te})$ from ab-initio theory: new direct band gap semiconductors, Eur. Phys. J. B 2012, 85, 186- 187.

[31.] Kadantsev, E. S.; Hawrylak, P. Electronic structure of a single $\mathrm{MoS}_{2}$ monolayer, Solid State Commun. 2012, 152, 909- 913.

[32.] Jin, W.; Yeh, P. C.; Zaki, N.; Zhang, D.; Sadowski, J. T.; Al-Mahboob, A.; van der Zande, A. M.; Chenet, D. A.; Dadap, J. I.; Herman, I. P. et al. Direct Measurement of the Thickness-Dependent Electronic Band Structure of MoS2 Using Angle-Resolved Photoemission Spectroscopy, Phys. Rev. Lett. 2013, 111, 106801.

[33.] Terrones, H.; Terrones, M. Ultralow thermal conductivity and high thermoelectric figure of merit in SnSe crystals, J. Mater. Res. 2014, 29, 373- 377.

[34.] Chen, Y.; Xi, J.; Dumcenco, D. O.; Liu, Z.; Suenaga, K.; Wang, D.; Shuai, Z.; Huang, Y. S.; Xie. L. Tunable Band Gap Photoluminescence from Atomically Thin Transition-Metal Dichalcogenide Alloys, ACS Nano 2013, 7, 4610- 4615.

[35.] Kosmider, K.; Fernandez-Rossier, Electronic properties of the MoS2-WS2 heterojunction, J. Phys. Rev. B2013, 87, 075451.

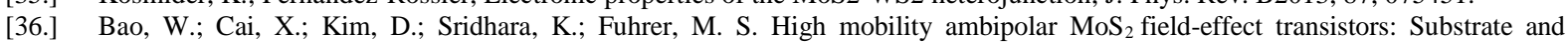
dielectric effects, Appl. Phys. Lett. 2013, 102, 042104.

[37.] Radisavljevic, B., Whitwick, M. B.; Kis, A. Integrated circuits and logic operations based on single-layer MoS2ACS Nano 2011, 5, 9934- 9938.

[38.] Wang, H.; Yu, L.; Lee, Y. H.; Shi, Y.; Hsu, A.; Chin, M. L.; Li, L. J.; Dubey, M.; Kong, J.; Palacios, T. Integrated circuits based on bilayer MoS2 transistors, Nano Lett. 2012, 12, 4674- 4680.

[39.] Radisavljevic, B.; Whitwick, M. B.; Kis, A. Small-signal amplifier based on single-layer MoS 2 , Appl. Phys. Lett. 2012, 101, 043103.

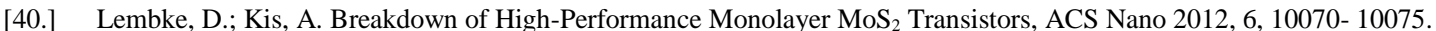

[41.] Kaul, A. B. Kaul, A. B. J. Mater. Res. 2014, 29, 348- 352, J. Mater. Res. 2014, 29, 348- 352.

[42.] Chhowalla, M.; Shin, H. S.; Eda, G.; Li, L. J.; Loh, K. P.; Zhang, H. The chemistry of two-dimensional layered transition metal dichalcogenide nanosheets, Nature Chemistry 2013, 5, 263 - 275.

[43.] Jariwala, D.; Sangwan, V. K.; Lauhon, L. J.; Marks, T. J.; Hersam, M. C. Emerging Device Applications for Semiconducting TwoDimensional Transition Metal Dichalcogenides, ACS Nano 2014, 8, 1102- 1120.

[44.] B. Bera, Literature Review on Electrospinning Process (A Fascinating Fiber Fabrication Technique), Imperial Journal of Interdisciplinary Research (IJIR) Vol-2, Issue-8, 2016. 
[45.] Y. Ahn, L. J.Young, H.Soon Man, L.Jaerock, H. Jongwook, H. C. Jin, Y. Seo, Enhanced Piezoelectric Properties of Electrospun Poly(Vinylidene fluoride)/Multiwalled Carbon Nanotube Composites due to High $\beta$-Phase Formation in Poly(Vinylidene fluoride). J. Phys. Chem. C 2013, 117, 11791-11799.

[46.] A. Splendiani, L.Sun, Y. Zhang, T. Li, J.Kim, Chim, Y.C., G.Galli, F.Wang Emerging Photoluminescence in Monolayer MoS2. Nano Lett. 2010, 10, 1271-1275.

[47.] S. Ruhle, M. Shalom and A. Zaban, Chem. Phys. Chem., 11 (2010) 2290.

[48.] L. Wang, L. Qin, and L. Li, "Piezoelectric dynamic pressure sensor," in Information and Automation (ICIA), 2010 IEEE International Conference on, 2010, pp. 906-911.

[49.] A. Kimoto and S. Shimada, "A Proposal of New Multifunctional Pressure Sensor Based on PVDF Films," IEEE Trans. Instrumentation and Measurement, , vol. 62, pp. 2870-2877, 2013.

[50.] S. Choi, Z. Jiang, "A novel wearable sensor device with conductive fabric and PVDF film for monitoring cardiorespiratory signals", Sens. Actuat. APhys., vol. 128, pp. 317-326, 2006.

[51.] K. Niizeki, I. Nishidate, K. Uchida, M. Kuwahara, "Unconstrained cardiorespiratory and body movement monitoring system for home care", Med. Biol. Eng. Comput., vol. 43, pp. 716-724, 2005

[52.] J. Siivola, "New noninvasive piezoelectric transducer for recording of respiration heart rate and body movements", Med. Biol. Eng. Comput., vol. 27, pp. 423-424, 1989.

[53.] kawai H. The piezoelectricity of polyvinyldene fluoride[J]. Journal of Applied Physics, 1969,8(7):975-976

[54.] Bauer, F Ferroelectric PVDF polymer for high pressure and shock compression sensors Electrets, 2002. ISE 11. Proceedings. 11th International Symposium Oct. 2002, 219-222.

[55.] Robert L. Clark, C.R. Fuller, Active Structural Acoustic Control with Adaptive Structures Including Wavenumber Considerations. The Journal of the Acoustical Society of America. 1992.

[56.] D.H. Kim, B. Kim, H. Kang. Development of a piezoelectric polymerbased sensorized microgripper for microassembly and micromanipulation[J],2004.

[57.] Wang Bor-Tsuen. The PVDF-based wave number domain sensing techniques for active sound radiation control from a simply supported beam, The Journal of the Acoustical Society of America, Volume 103, Issue 4, April 1998, pp.1904-1915. 